\title{
Ulmus minor bark hydro-alcoholic extract ameliorates histological parameters and testosterone level in an experimental model of PCOS rats
}

\author{
Mohammad Javad Hoseinpour ${ }^{1}$, Alireza Ghanbari ${ }^{1}$, Nahid AzaD ${ }^{2}$, Ali Zare ${ }^{1}$, Shabnam Abdi ${ }^{3}$, \\ Ensieh SaJadi ${ }^{4}$, Hojjat-Allah AbbaszadeH ${ }^{4}$, Reza Mastery Farahani ${ }^{4}$, Mohammad-Amin Abdollahifar ${ }^{4}$ \\ ${ }^{1}$ Young Researchers and Elite Club, Islamic Azad University, Tehran Medical Sciences Branch, Tehran, Iran; ${ }^{2}$ Abnormal \\ Uterine Bleeding Research Center, Semnan University of Medical Sciences, Semnan, Iran; ${ }^{3}$ Department of Anatomical \\ Sciences \& Cognitive Neuroscience, Faculty of Medicine, Tehran Medical Sciences, Islamic Azad University, Tehran, Iran; \\ ${ }^{4}$ Department of Biology and Anatomical Sciences, School of Medicine, Shahid Beheshti University of Medical Sciences, \\ Tehran, Iran \\ E-mail: abdollahima@sbmu.ac.ir; realmastery@hotmail.com
}

\begin{abstract}
Objective. Polycystic ovary syndrome (PCOS) is a common and multifactorial disease associated with female factor infertility. Ulmus minor bark (UMB) is one of the medicinal plants used in Persian folklore as a fertility enhancer. In the current study, we aimed to elucidate the effect of UMB hydro-alcoholic extract on histological parameters and testosterone condition in an experimental model of PCOS rats.

Methods. Thirty female rats were randomly divided into five groups: (1) control, (2) vehicle, (3) PCOS $/ 50 \mathrm{mg}[6 \mathrm{mg} / \mathrm{kg}$ dehydroepiandrosterone (DHEA) $+50 \mathrm{mg} / \mathrm{kg}$ UMB hydro-alcoholic extract], (4) PCOS/150 mg (6 mg/kg DHEA + $150 \mathrm{mg} / \mathrm{kg}$ UMB hydro-alcoholic extract), and (5) PCOS (6 mg/kg DHEA). All interventions were performed for 21 days. Afterwards, stereological analysis was done for determination of ovarian volume and follicle number. The serum level of testosterone was measured by ELISA kit.

Results. UMB hydro-alcoholic extract improved the total number of the corpus luteum in the treatment groups when compared to the PCOS group ( $<<0.05)$. PCOS/150 mg and PCOS/50 mg groups showed significantly lower total number of the primordial, primary, and secondary follicles as well as testosterone level compared to the PCOS group $(\mathrm{p}<0.05)$. The total number of antral follicles and volume of ovary did not differ significantly between groups.

Conclusion. UMB extract may be an effective and good alternative in improving PCOS histological and testosterone disturbances although further studies are warranted to confirm the safety of UMB plant in human.
\end{abstract}

Key words: ovary, PCOS, testosterone, stereology, ulmus minor bark

Infertility is one of the problems, which many couples all over the world suffer from and causes a lot of stress and losing the foundation of the family (Tao et al. 2011; O’Flynn 2014). Smoking, overweight or underweight, infections, consumption of chemical foods and drugs and activity in industrial environ- ments, which are collectively named as "life style" may affect female fertility (Anderson et al. 2010). The polycystic ovary syndrome (PCOS) is a metabolic and based endocrinopathy lifestyle disorder affecting the fertility in females. It is characterized by a combination of signs and symptoms such as anovulation or

Corresponding authors: Mohammad-Amin Abdollahifar, Department of Biology and Anatomical Sciences, School of Medicine, Shahid Beheshti University of Medical Sciences, Tehran, Iran; phone: +98-2122439976; fax: +98-2122439976;

e-mail: m_amin58@yahoo.com, abdollahima@sbmu.ac.ir. Reza Mastery Farahani, Department of Biology and Anatomical

Sciences, School of Medicine, Shahid Beheshti University of Medical Sciences, Tehran, Iran; phone: +98-2122439976; fax: +982122439976; e-mail: realmastery@hotmail.com. 
oligovulation, hyperandrogenism, hyperinsulinism, hirsutism, seborrhea, and etc. (Escobar-Morreale 2018; Patel 2018).

Many medicinal plants are recommended by tradipractitioners for reproductive health issues in different countries (Adnan et al. 2015; Ong and Kim 2015; Yemele et al. 2015; Yazbek et al. 2016). Based on the ethnopharmacological studies, valuable results have been reported regarding to the application of medicinal herbs in the treatment of infertility, which, of course, requires further investigations. It is important to know more about the pharmacological properties of medicinal plants used in the traditional medicine (Telefo et al. 2011; van Andel et al. 2014). So far, various herbal remedies have been used for ameliorate signs and symptoms of PCOS as recommended by traditional medicine (Ried 2015; Ma and Tan 2017; Hosseinkhani et al. 2018).

Ulmus minor bark (UMB) is one of the medicinal plants used in Persian folklore as a female fertility enhancer. In western province of Iran, including Lorestan and Kermanshah, elm bark is abundantly use in the treatment of female infertility. In the terms of epidemiology, the benefits of its application in female infertility treatment have been reported (Bahmani et al. 2016). According to our knowledge, possible beneficial and adverse effects of elm bark on the female fertility have not studied yet scientifically. Considering the fact that PCOS is a common disorder among infertile women, while there is still no definite treatment for it, we designed the present study to evaluate the effect of UMB hydro-alcoholic extract on follicular parameters and testosterone level in an experimental model of PCO rats.

\section{Material and methods}

Plant material and preparation of the extract. Ulmus minor bark was harvested from Lorestan (western of Iran). After cleaning, the powdered of leaves were soaked on $70 \%$ ethanol at room temperature with occasional shaking for $72 \mathrm{~h}$, after the filtrated mixture dried at room temperature to preparation powder and stored at $4^{\circ} \mathrm{C}$ for all experiments.

Animals. In this experimental study, thirty female rats were randomly divided into five groups $(n=6)$ : (1) control, (2) vehicle, (3) PCOS/50 mg (6 mg/kg dehydroepiandrosterone, DHEA + $50 \mathrm{mg} / \mathrm{kg} \mathrm{UMB}$ hydro-alcoholic extract), (4) PCOS/150 mg (6 mg/kg $\mathrm{DHEA}+150 \mathrm{mg} / \mathrm{kg}$ UMB hydro-alcoholic extract), and (5) PCOS (6 mg/kg DHEA). UMB hydro-alcoholic extract dissolved in normal saline and injected for 21 days. The animals were housed under standard conditions, room temperature $\left(22-24^{\circ} \mathrm{C}\right), 12: 12 \mathrm{~h}$ light-dark schedule and free access to water and food.

PCOS model. As the study model, female rats of the PCOS group were injected with DHEA (6 mg/kg body weight, dissolved in $0.05 \mathrm{ml}$ sesame oil), daily for 21 days (Oakley et al. 2011).

The estrus synchronization. In order to synchronize the estrus cycles of the rats, $0.5 \mu \mathrm{g}$ of cloprostenol acetate was injected intraperitoneally on the first day and after 3 days, $3 \mu \mathrm{g}$ of progesterone was injected subcutaneously.

Blood sampling and testosterone measurement. The blood samples for hormonal measurement were obtained after deep anesthesia from the heart. Blood samples were centrifuged at $6000 \mathrm{~g}$ for 5 minutes at $4^{\circ} \mathrm{C}$ and then stored at $-80^{\circ} \mathrm{C}$ until use. Rat specific ELISA kit was used to measure blood serum level of testosterone (catalogue no. CSB-E11162r).

Tissue preparation. At the end of the experiment, the rats were anesthetized and their ovaries were removed and fixed in $4 \%$ neutral buffered formalin. After tissue processing, the samples were embedded into paraffin blocks. Then, complete serial sections (10 $\mu \mathrm{m}$ thick) using a microtome were prepared. About 10 sections in each animal were selected in a systematic random manner. The sections were stained with Hematoxylin and Eosin (H\&E).

Stereological study.

Volume of ovary. The Cavalieri method was applied to estimate the total volume of the ovary, cortex, and medulla (Gundersen et al. 1988a,b). The systematic random sections were sampled and the volume was estimated using the following formula:

$$
V=\sum P \times t \times \frac{a}{p}
$$

In this formula, $\Sigma \mathrm{P}, \mathrm{t}$, and $\mathrm{a} / \mathrm{p}$ are the total number of points superimposed on the images, the distance between the sections, and the area associated with each point, respectively.

Number of primordial, primary, secondary, antral follicles, and corpus luteum. The number of primordial, primary, secondary, antral follicles, and corpus luteum was estimated using a Nikon E200 microscope (Nikon, Tokyo, Japan), which was connected with a camera. The microscopic fields were selected through systematic uniform random sampling by moving the microscope stage at the same distance each time to estimate the number of follicles and corpus luteum (Gundersen et al. 1988a,b). An optical dissector design was used to sample the tissue in conjunction with an unbiased sampling. Numerical density $(\mathrm{Nv})$ wascalculated with the following formula: 


$$
N_{v}=\frac{\sum Q}{\sum P \times h \times \frac{a}{f}} \times \frac{t}{B A}
$$

Where " $\Sigma \mathrm{Q}$ " is the number of the nuclei, " $\Sigma \mathrm{P}$ " is the total number of the unbiased counting frame in all fields, " $h$ " is the height of the dissector, "a/f" is the frame area, " $\mathrm{t}$ " is the real section thickness measured in every field using the microcator, and "BA" is the block advance of the microtome, which was set at $10 \mu \mathrm{m}$. The total number of primordial, primary, secondary, antral follicles, and corpus luteum was estimated by multiplying the numerical density $(\mathrm{Nv})$ by the total $\mathrm{V}(20)$.

$$
N_{\text {total }}=N_{v} \times V
$$

Statistical analysis. The results were analyzed by one-way ANOVA analysis of variance and Tukey's test. The means were considered significantly different if $\mathrm{p}<0.05$. Data are shown as mean $\pm \mathrm{SD}$.

\section{Results}

Serum testosterone condition. Evaluation of testosterone indicated that the level of testosterone hormone in PCOS group was significantly higher compared to the control (140.1 vs. $24.5 \mathrm{ng} / \mathrm{dl})$ and sham $(140.1$ vs. $25.6 \mathrm{ng} / \mathrm{dl})$ groups $(\mathrm{p}<0.05)$. We also found that the level of testosterone hormone decreased significantly in $\mathrm{PCOS} / 50 \mathrm{mg}$ (54.9 vs. $140.1 \mathrm{ng} / \mathrm{dl}$ ) and PCOS/150 mg (72 vs. $140.1 \mathrm{ng} / \mathrm{dl})$ groups in comparison with the PCOS group $(\mathrm{p}<0.05)$ (Figure 1).

Total volume of ovary. Our results showed that the volume of ovary, cortex, and medulla did not differ between control, sham, PCOS, and UMB hydro-alcoholic extract treated groups significantly (Figure 2).

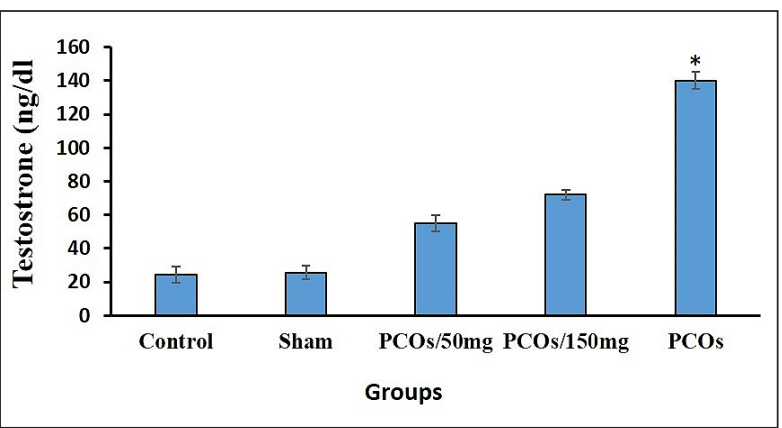

Figure 1. The level of testosterone in different groups. PCOS group showed a significant difference with the control, sham, PCOS/50 mg and PCOs/150 mg groups. The level of testosterone also showed a significant difference between PCOS/50 mg with PCOS/150mg and PCOS. ${ }^{*} \mathrm{p}<0.05$.
Total number of primordial, primary, secondary, antral follicles, and corpus luteum. Total number of the primordial, primary, and secondary follicles was significantly higher in PCOS group compared to the control and sham groups $(\mathrm{p}<0.05)$. We found that the total number of that follicles decreased in PCOS/50 $\mathrm{mg}$ group compared to the PCOS group $(\mathrm{p}<0.05)$. Although there was no significant difference between PCOS/150 mg and PCOS groups with respect to follicle number (Figure 3 and Figure 5).

As shown in Figure 3, the total number of antral follicles did not differ significantly between groups. The results also revealed that the number of corpus luteum in UMB hydro-alcoholic extract treated groups were significantly increased compared to the PCOS group $(\mathrm{p}<0.05)$ (Figure 4 and Figure 5).

\section{Discussion}

In the present study, we evaluated the effects of UMB hydro-alcoholic extract on follicular parameters and testosterone conditions in an experimental

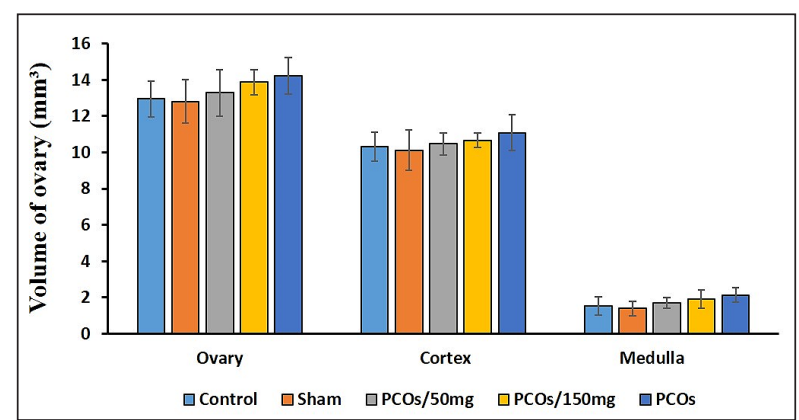

Figure 2. The total volume of ovary, cortex and medulla in the different groups. There was no significant difference between groups.

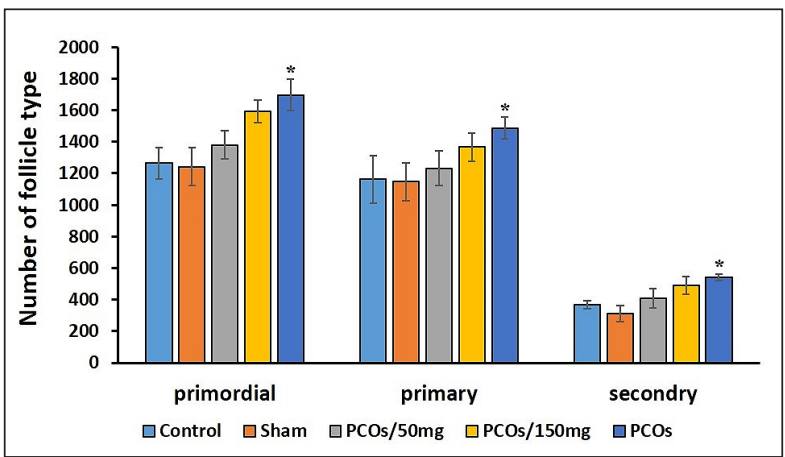

Figure 3. The total number of primordial, primary, and secondary follicles in the different groups. PCOS group showed a significant difference with the control, sham, and PCOS/50 mg groups. The total number of primordial, primary, and secondary follicles also showed a significant difference between $\mathrm{PCOS} / 50 \mathrm{mg}, \mathrm{PCOS} / 150 \mathrm{mg}$ and PCOS. ${ }^{*} \mathrm{p}<0.05$. 
model of PCOS female rats and observed its useful properties on undesirable signs of PCOS.

PCOS is a disorder affecting fertility in females via its criteria such as anovulation or oligovulation, hyperandrogenism, decreased follicular development, increased ovarian volume and etc. (EscobarMorreale 2018; Patel 2018). Abnormalities in the early stages of folliculogenesis may be one of the main causes of ovulation failure in PCOS (Franks et al. 2008). Increased number of immature follicles in ovary from patients with PCOS have been reported before (Maciel et al. 2004; Franks et al. 2008). Other manifestations of the disease are visceral obesity, increased body fat, and diabetes mellitus (Yildirim et al. 2003). The mechanisms that cause PCOS are very diverse and complex. Inflammatory biomarkers such as tumor necrosis factor- $\alpha$ (TNF- $\alpha$ ), intercellular adhesion molecule-1 (ICAM-1), the mean platelet volume (MPV), serum IL-6, and monocyte chemoattractant protein-1 (MCP-1) are higher in PCOS patients compared to control women (Dasanu et al. 2011; Yilmaz et al. 2015; Peng et al. 2016). Adipocytokines levels such as leptin, Omentin-1 and chemerin have been reported to be altered in patients with PCOS. Hormonal disturbances, including increased level of androgens, estradiol, AMH and lower level of progesterone, also are one of etiologies related to PCOS (Patel 2018). Oxidative stress is another cause contributing in PCOS formation. The sources of the oxidative stress in PCOS are the presence of excessive adipose tissue, fatty acid oxidation, mitochondrial

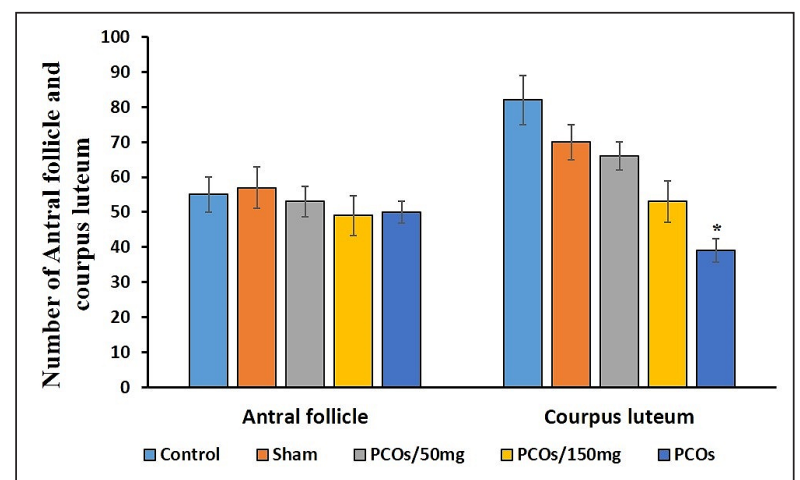

Figure 4. The total number of antral follicles and corpus luteum in the different groups. There was a significant difference between PCOS and other groups regarding with the number of corpus luteum. ${ }^{\star} \mathrm{p}<0.05$.

dysfunction, and increased activity of ROS and nitric oxide producing enzymes (Macut et al. 2013).

DHEA application ranged $1.5-6 \mathrm{mg} / \mathrm{kg}$ can cause appearing hormonal and histological features of PCOS in animals (Roy et al. 1962). Disturbed ovulation, corpus luteum defects, increased atretic follicles, and hyperandrogenism are some of induced disturbances following DHEA application in rodents (McNeilly and Duncan 2013). In the present study, we observed hyperandrogenism, decreased number of corpus luteum, and increased number of primordial, primary and secondary follicles in PCOS model of rats after DHEA application.

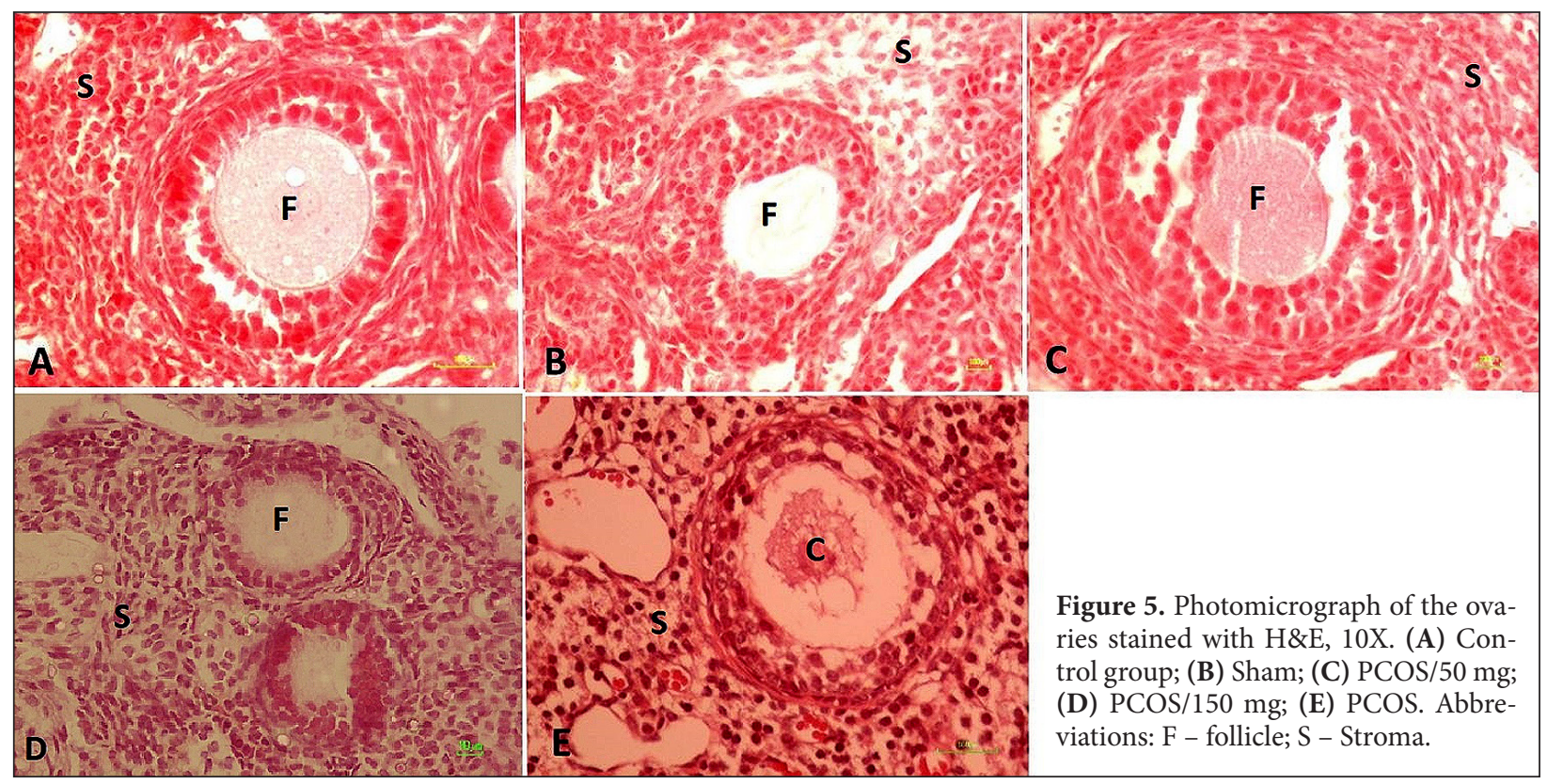


$\mathrm{UMB}$ is the bark of elm tree. Elm tree is a flowering plant originating in current central Asia. In western province of Iran, narvan elm is cultivated as a shade tree and is recommended by traditional medicine practitioners for infertility treatment in females (Bahmani et al. 2016). Separation and identification of Elm bark components using gas chromatography/ mass spectrometry methods was performed by Martin et al. (2004). They mainly identified aliphatic hydrocarbons (particularly heptacosane and nonacosane) in current-year twigs and triterpenes in older stems, although a variation was observed between samples gathered in different locations. The main components recognized in UMB was lupenol (lupeol), $\beta$-sitosterol, and hydroxycoamarins, especially scopoletin, and isofraxidin (Martin et al. 2004).

Triterpenes are members of the phytosterol family that found widely in different parts of plants. Lupeol, a member of triterpenes, identified in elm bark with quantity of $800 \mu \mathrm{g} / \mathrm{g}$. Some beneficial effects of lupeol are anti-inflammatory, antioxidant, anti-microbial, anti-cancer, and cell proliferative properties (Siddique and Saleem 2011; Agra et al. 2015). The effects of lupeol on PCOS has been investigated previously by Rezaei-Golmisheh et al. (2017). In that study, lupeol decreased the endometrial hyperfibrosis and hyperplasia was induced by DHEA (Rezaei-Golmisheh et al. 2017). The reason for such outcome may be related to the role of lupeol as an androgen receptor inhibitor reported previously (Siddique et al. 2011). Other phytopharmacological extract in the bark of elm tree is $\beta$-sitosterol, which has a structure and function similar to cholesterol and is referred as "key of life". Solubility of $\beta$-sitosterol is low that restricts its use (AbuMweis et al. 2014; Yin et al. 2018). Based on the best available evidence in literature, various pharmacological properties for phytosterol have been studied (Wilt et al. 1999; Shin et al. 2018; Yin et al. 2018). $\beta$-sitosterol applies its anti-tumor properties via MPK/PTEN/HSP90 axis in AGS human gastric adenocarcinoma cells (Shin et al. 2018). Antioxidant and anti-inflammatory effects of $\beta$-sitosterol derivatives on acute hepatic injury was reported by Yin et al. (2018). In addition, treatment with $\beta$-sitosterol in patients with benign prostatic hyperplasia improves urinary flow measure which may be due to antiinflammatory properties or cholesterol metabolism (Wilt et al. 1999). As we know, one of the symptoms of the PCOS is hyperandrogenism. Interestingly, it has been shown that $\beta$-sitosterol inhibits the conversion of testosterone to dihydrotestostrone, thereby improving some symptoms of PCO which caused by dehydrotestostrone (Klippel et al. 1997; Dunne and
Slater 2005). Hydroxycoamarins especially scopoletin and isofraxidin are also other compounds identified in elm bark albeit in lower concentrations (Martin et al. 2004). Some of beneficial effects of isofraxidin have been reported to be inhibition of pro-inflammatory cytokines (Liu et al. 2015), inhibition of lipid production and inflammation in the liver (Li et al. 2017), anti-oxidant and anti-bacterial properties (Khan et al. 2009). Scopoletin is a coumarin (7-hydroxy 6-methoxycoumarin) compound which, based on previous studies, has beneficial effects such as antioxidant (Shaw et al. 2003), anti-tumoral (Liu et al. 2012), anti-inflammatory (Ding et al. 2008; Leema and Tamizhselvi 2018), hypoglycemic and hypolipidemic activity (Verma et al. 2013). Based on ethnopharmacological studies, various medicinal herbs have been applied for ameliorate signs and symptoms of PCO. Green tea (Ghafurniyan et al. 2015), citrullus colocynthis (Barzegar et al. 2017), grape seed (Salmabadi et al. 2017), and trigonella foenum-graecum seed extract (Swaroop et al. 2015) are samples of herbal remedies which have been used in treatment of PCOS and reported beneficial results. Additionally, in traditional Chinese medicine, the combination of several plants is used to treat patients with PCO (Ried 2015). Similarly, we applied UMB hydro-alcoholic extract in a PCOS model of rat and observed its beneficial effects on testosterone level and histological disturbances. We showed that UMB hydro-alcoholic extract improved the number of corpus luteum in the treatment groups. The number of follicles in different stages as well as the level of testosterone were decreased in UMB treated groups. Although the number of antral follicles and ovarian volume showed no significant difference between groups. We observed no adverse effects in UMBtreated groups compared to the other groups with respect to histological and hormonal parameters.

As previously mentioned, the various compounds in the ulmus minor bark have antioxidant effects (lupeol, $\beta$-sitosterol, isofraxidin, scopoletin), antiinflammatory properties (lupeol, $\beta$-sitosterol, isofraxidin, scopoletin), ability to lipid production inhibition (isofraxidin, scopoletin), hypoglycemic activities (scopoletin), and also anti-androgenic role (lupeol). It seems that the positive effects observed in this study are probably due to the therapeutic effects of these compounds. It should be noted that the lower dose $(50 \mathrm{mg}$ ) of the UMB extract reduced the volume of ovary, cortex, and medulla more than its higher dose (150 mg), although the difference was not significant. Similar results were observed for the two other parameters (testosterone level and primor- 
dial, primary, and secondary follicle numbers). In addition, the dose of $50 \mathrm{mg}$ increased the number of corpus luteum more than the $150 \mathrm{mg}$ dose. Indeed, we observed that lower dose of UMB extract $(50 \mathrm{mg})$ was more effective compared to upper dose $(150 \mathrm{mg})$ in alleviating hyperandrogenism and histological disturbances in PCOS model. We concluded that excessive consumption of this plant has no further therapeutic effect and must be used more cautiously in PCOS women. To the best our knowledge, we showed for the first time that UMB extract may be an effective and good alternative in improving PCOS histological and hormonal disturbances, although further studies are warranted to confirm the safety of UMB plant and its proper dose in women with PCOS.

\section{Acknowledgment}

The present work was carried out at the Department of Anatomical Sciences and Biology, School of Medicine, Shahid Beheshti University of Medical Sciences, Tehran, Iran.

\section{References}

AbuMweis SS, Marinangeli CP, Frohlich J, Jones PJ. Implementing phytosterols into medical practice as a cholesterollowering strategy: overview of efficacy, effectiveness, and safety. Can J Cardiol 30, 1225-1232, 2014.

Adnan M, Tariq A, Mussarat S, Begum S, AbdEIsalam NM, Ullah R. Ethnogynaecological assessment of medicinal plants in Pashtun's tribal society. Biomed Res Int 2015, 196475, 2015.

Agra LC, Ferro JN, Barbosa FT, Barreto E. Triterpenes with healing activity: A systematic review. J Dermatolog Treat 26, 465-470, 2015.

Anderson K, Norman RJ, Middleton P. Preconception lifestyle advice for people with subfertility. Cochrane Database Syst Rev 4, CD008189, 2010.

Bahmani M, Baharvand-Ahmadi B, Tajeddini P, Rafieian-Kopaei M, Naghdi N. Identification of medicinal plants for the treatment of kidney and urinary stones. J Renal Inj Prev 5, 129-133, 2016.

Barzegar MH, Khazali H, Kalantar SM, Khoradmehr A. Effect of Citrullus colocynthis hydro-alcoholic extract on hormonal and folliculogenesis process in estradiol valerate-induced PCOs rats model: An experimental study. Int J Reprod Biomed (Yazd) 15, 661-668, 2017.

Dasanu CA, Clark BA, Ichim TE, Alexandrescu DT. Polycystic ovary syndrome: focus on platelets and prothrombotic risk. South Med J 104, 174-178, 2011.

Ding Z, Dai Y, Hao H, Pan R, Yao X, Wang Z. Anti-Inflammatory Effects of scopoletin and underlying mechanisms. Pharmaceutical Biology 46, 854-860, 2008.

Dunne N, Slater B. The Natural Diet Solution for PCOS and Infertility. Health Solutions Press, 2005.

Escobar-Morreale HF. Polycystic ovary syndrome: definition, aetiology, diagnosis and treatment. Nat Rev Endocrinol 14, 270-284, 2018.

Franks S, Stark J, Hardy K. Follicle dynamics and anovulation in polycystic ovary syndrome. Hum Reprod Update 14, 367-378, 2008.

Ghafurniyan H, Azarnia M, Nabiuni M, Karimzadeh L. The effect of green tea extract on reproductive improvement in estradiol valerate-induced polycystic ovarian syndrome in rat. Iran J Pharm Res 14, 1215-1233, 2015.

Gundersen HJ, Bendtsen TF, Korbo L, Marcussen N, Moller A, Nielsen K, Nyengaard JR, Pakkenberg B, Sorensen FB, Vesterby A, West MJ. Some new, simple and efficient stereological methods and their use in pathological research and diagnosis. APMIS 96, 379-394, 1988a.

Gundersen HJ, Bagger P, Bendtsen TF, Evans SM, Korbo L, Marcussen N, Moller A, Nielsen K, Nyengaard JR, Pakkenberg B, Sorensen FB, Vesterby A, West MJ. The new stereological tools: disector, fractionator, nucleator and point sampled intercepts and their use in pathological research and diagnosis. APMIS 96, 857-881, 1988b.

Hosseinkhani A, Asadi N, Pasalar M, Zarshenas MM. Traditional Persian Medicine and management of metabolic dysfunction in polycystic ovary syndrome. J Tradit Complement Med 8, 17-23, 2018.

Khan S, Riaz N, Afza N, Malik A, Aziz-ur-Rehman, Iqbal L, Lateef M. Antioxidant constituents from Cotoneaster racemiflora. J Asian Nat Prod Res 11, 44-48, 2009.

Klippel KF, Hiltl DM, Schipp B. A multicentric, placebo-controlled, double-blind clinical trial of beta-sitosterol (phytosterol) for the treatment of benign prostatic hyperplasia. German BPH-Phyto Study group. Br J Urol 80, 427-432, 1997.

Leema G. Tamizhselvi R. Protective effect of scopoletin against cerulein-induced acute pancreatitis and associated lung injury in mice. Pancreas 47, 577-585, 2018. 
Li J, Li X, Li Z, Zhang L, Liu Y, Ding H, Yin S. Isofraxidin, a coumarin component improves high-fat diet induced hepatic lipid homeostasis disorder and macrophage inflammation in mice. Food Funct 8, 2886-2896, 2017.

Liu W, Hua J, Zhou J, Zhang H, Zhu H, Cheng Y, Gust R. Synthesis and in vitro antitumor activity of novel scopoletin derivatives. Bioorg Med Chem Lett 22, 5008-5012, 2012.

Liu L, Mu Q, Li W, Xing W, Zhang H, Fan T, Yao H, He L. Isofraxidin protects mice from LPS challenge by inhibiting pro-inflammatory cytokines and alleviating histopathological changes. Immunobiology 220, 406-413, 2015.

Ma QW, Tan Y. Effectiveness of co-treatment with traditional Chinese medicine and letrozole for polycystic ovary syndrome: a meta-analysis. J Integr Med 15, 95-101, 2017.

Maciel GA, Baracat EC, Benda JA, Markham SM, Hensinger K, Chang RJ, Erickson GF. Stockpiling of transitional and classic primary follicles in ovaries of women with polycystic ovary syndrome. J Clin Endocrinol Metab 89, 5321-5327, 2004.

Macut D, Bjekic-Macut J, Savic-Radojevic A. Dyslipidemia and oxidative stress in PCOS. Front Horm Res 40, 51-63, 2013.

Martin D, Garcia-Vallejo MC, Pajares JA, Lopez D, Diez JJ. Elm bark components and their potential influence on bark beetle feeding. Forest Systems 13, 227-235, 2004.

McNeilly AS, Duncan WC. Rodent models of polycystic ovary syndrome. Mol Cell Endocrinol 373, 2-7, 2013.

O’Flynn N. Assessment and treatment for people with fertility problems: NICE guideline. Br J Gen Pract 64, 50-51, 2014.

Oakley O, Lin PC, P Bridges P, Ko C. Animal models for the study of polycystic ovarian syndrome. Endocrinology and Metabolism 26, 6193-6202, 2011.

Ong HG, Kim YD. Herbal therapies and social-health policies: Indigenous Ati Negrito women's dilemma and reproductive healthcare transitions in the Philippines. Evid Based Complement Alternat Med 2015, 491209, 2015.

Patel S. Polycystic ovary syndrome (PCOS), an inflammatory, systemic, lifestyle endocrinopathy. J Steroid Biochem Mol Biol 182, 27-36, 2018.

Peng Z, Sun Y, Lv X, Zhang H, Liu C, Dai S. Interleukin-6 levels in women with polycystic ovary syndrome: A Systematic review and meta-analysis. PLoS One 11, e0148531, 2016.

Rezaei-Golmisheh A, Sadrkhanlou RA, Malekinejad H, Ahmadi A. The ameliorative effects of lupeol and flutamide on dehydroepiandrosterone-induced uterine hyperfibrosis in mice. The Journal of Urmia University of Medical Sciences 26, 923-934, 2017.

Ried K. Chinese herbal medicine for female infertility: an updated meta-analysis. Complement Ther Med 23, 116$128,2015$.

Roy S, Mahesh VB, Greenblatt RB. Effect of dehydroepiandrosterone and delta4-androstenedione on the reproductive organs of female rats: production of cystic changes in the ovary. Nature 196, 42-43, 1962.

Salmabadi Z, Mohseni Kouchesfahani H, Parivar K, Karimzadeh L. Effect of grape seed extract on lipid profile and expression of interleukin-6 in polycystic ovarian syndrome Wistar rat model. Int J Fertil Steril 11, 176-183, 2017.

Shaw CY, Chen CH, Hsu CC, Chen CC, Tsai YC. Antioxidant properties of scopoletin isolated from Sinomonium acutum. Phytother Res 17, 823-825, 2003.

Shin EJ, Choi HK, Sung MJ, Park JH, Chung MY, Chung S, Hwang JT. Anti-tumour effects of beta-sitosterol are mediated by AMPK/PTEN/HSP90 axis in AGS human gastric adenocarcinoma cells and xenograft mouse models. Biochem Pharmacol 152, 60-70, 2018.

Siddique HR, Saleem M. Beneficial health effects of lupeol triterpene: a review of preclinical studies. Life Sci 88, 285-293, 2011.

Siddique HR, Mishra SK, Karnes RJ, Saleem M. Lupeol, a novel androgen receptor inhibitor: implications in prostate cancer therapy. Clin Cancer Res 17, 5379-5391, 2011.

Swaroop A, Jaipuriar AS, Gupta SK, Bagchi M, Kumar P, Preuss HG, Bagchi D. Efficacy of a movel fenugreek seed extract (Trigonella foenum-graecum, Furocyst) in polycystic ovary syndrome (PCOS). Int J Med Sci 12, 825-831, 2015.

Tao P, Coates R, Maycock B. The impact of infertility on sexuality: A literature review. Australas Med J 4, 620-627, 2011.

Telefo PB, Lienou LL, Yemele MD, Lemfack MC, Mouokeu C, Goka CS, Tagne SR, Moundipa FP. Ethnopharmacological survey of plants used for the treatment of female infertility in Baham, Cameroon. J Ethnopharmacol 136, 178-187, 2011.

van Andel T, de Boer HJ, Barnes J, Vandebroek I. Medicinal plants used for menstrual disorders in Latin America, the Caribbean, sub-Saharan Africa, South and Southeast Asia and their uterine properties: A review. J Ethnopharmacol 155, 992-1000, 2014. 
Verma A, Dewangan P, Kesharwani D, Kela SP. Hypoglycemic and hypolipidemic activity of scopoletin (coumarin derivative) in streptozotocin induced diabetic rats. Int J Pharm Sci Rev Res 22, 79-83, 2013.

Wilt TJ, MacDonald R, Ishani A. beta-sitosterol for the treatment of benign prostatic hyperplasia: a systematic review. BJU Int 83, 976-983, 1999.

Yazbek PB, Tezoto J, Cassas F, Rodrigues E. Plants used during maternity, menstrual cycle and other women's health conditions among Brazilian cultures. J Ethnopharmacol 179, 310-331, 2016.

Yemele MD, Telefo PB, Lienou LL, Tagne SR, Fodouop CS, Goka CS, Lemfack MC, Moundipa FP. Ethnobotanical survey of medicinal plants used for pregnant women's health conditions in Menoua division-West Cameroon. J Ethnopharmacol 160, 14-31, 2015.

Yildirim B, Sabir N, Kaleli B. Relation of intra-abdominal fat distribution to metabolic disorders in non-obese patients with polycystic ovary syndrome. Fertil Steril 79, 1358-1364, 2003.

Yilmaz O, Calan M, Kume T, Temur M, Yesil P, Senses MY. The effect of prolactin levels on MPV in women with PCOS. Clin Endocrinol (Oxf) 82, 747-752, 2015.

Yin Y, Liu X, Liu J, Cai E, Zhu H, Li H, Zhang L, Li P, Zhao Y. Beta-sitosterol and its derivatives repress lipopolysaccharide/d-galactosamine-induced acute hepatic injury by inhibiting the oxidation and inflammation in mice. Bioorg Med Chem Lett 28, 1525-1533, 2018. 